\title{
INFRA-ESTRUTURA PORTUÁRIA NACIONAL DE APOIO AO COMÉRCIO EXTERIOR: HIERARQUIZAÇÃO DAS UNIDADES PORTUÁRIAS
}

\author{
Wanda Fritsch da Silva e Souza ${ }^{1}$ \\ Geraldo da Silva e Souza ${ }^{2}$
}

\begin{abstract}
Resumo: Com a utilização de Componentes Principais derivam-se índices lineares multidimensionais com o intuito de avaliar os portos brasileiros quanto a sua importância relativamente às características de Oferta de Infra-estrutura, Resultados Operacionais, Aspectos Geo-econômicos e Demanda Projetada para o ano de 2011. Os postos (ranks) normalizados pelo máximo dos escores dos portos nesses construtos são utilizados, como insumos e produtos, em modelos de Análise de Envelopamento de Dados (DEA), com características de avaliação multicritério, na determinação de uma ordenação de importância dos portos brasileiros. Tal ordenação é original, tem base não subjetiva e pode servir ao propósito de determinação de prioridades na alocação de recursos nas unidades portuárias, no contexto de seu suporte ao comércio exterior brasileiro.
\end{abstract}

Palavras-chave: Portos Brasileiros, Análise Multivariada, Análise de Envelopamento de Dados.

\begin{abstract}
We use Principal Components to derive linear indices with the objective to evaluate the importance of Brazilian ports in regard to the characteristics of InfraStructure, Operational Results, Geo-economic Aspects, and Projected Demand for the year 2011. Ranks, normalized by the maximum rank, of the port scores in each of those constructs are used as inputs and outputs in Data Envelopment Analysis models of multicriteria evaluation to classify the Brazilian ports according their importance. This ordination is original, has a non subjective basis and may be used to set priorities in the context of resource allocations in the context of the ports support to the Brazilian foreign trade.
\end{abstract}

Key-words: Brazilian Ports, Multivariate Analysis, Data Envelopment Analysis.

\section{INTRODUÇÃO}

A hierarquização das unidades portuárias, de que trata este artigo, é requisito indispensável para o planejamento estratégico da infraestrutura nacional de apoio ao comércio exterior.

Neste artigo visa-se identificar os portos onde deve haver uma concentração de recursos orçamentários para investimentos que resultem em

\section{acréscimos}

movimentação

significativos

na conseqüentes o setor e que venham a contribuir para a melhoria e ampliação da corrente de comércio.

Os métodos tradicionais de hierarquização de importância utilizados nas áreas de economia, pesquisa operacional e psicometria baseiam-se tipicamente em avaliações subjetivas. Alguns exemplos dessas técnicas na

\footnotetext{
${ }^{1}$ Centro de Excelência em Engenharia de Transportes - CENTRAN, wandasouza@solar.com.br

${ }^{2}$ Universidade de Brasília - UnB, Departamento de Estatística, geraldosouza@unb.br
} 
literatura são definidos pelo Processo Hierárquico Analítico de Saaty (Saaty, 1994), pela Lei dos Julgamentos Categóricos de Thurstone (Souza, 2002) e DEA (Cook, 2004). Neste artigo utilizase uma ótica diferente dessas abordagens em que se combina uma técnica de Análise Multivariada - Componentes Principais, com a análise DEA - Data Envelopment Analysis, para produzir uma hierarquização. A classificação tem propriedades ótimas em dois níveis. Maximiza a dispersão em cada dimensão, via a Análise de Componentes Principais e o escore relativo de cada porto, visto como indutor e receptor de "desenvolvimento", via o DEA multicritério. A utilização de DEA em modelos de percepção de importância não é nova e pode ser vista em Thompson, Singleton e Thrall, (1986), Lovell e Pastor (1999), De Koeijer et al (2002), Cook (2004) e Leta et al (2005). Nossa contribuição para essa literatura consiste em combinar o DEA multicritério com componentes principais para a classificação dos portos brasileiros. A aplicação no contexto de portos é original no nível de nosso conhecimento.

A exposição procede como segue. $\mathrm{Na}$ Seção 2 listam-se os portos objeto da análise e as variáveis estudadas. $\mathrm{Na}$ Seção 3 descrevem-se os aspectos metodológicos. Na Seção 4 apresentamse os resultados da classificação e finalmente na Seção 5 um resumo e as conclusões do estudo.

\section{POPULAÇÃO DE PORTOS}

Na primeira fase deste trabalho foram definidos os portos a serem avaliados. Sendo assim, foram considerados os portos marítimos que dispunham de dados imprescindíveis para 0 desenvolvimento do estudo. Os terminais de uso privativo não foram considerados neste conjunto uma vez que o objetivo do estudo é a alocação de recursos oriundos do orçamento federal.

Depois de acurada análise da rede de portos marítimos organizados de uso público existentes no País, para efeito da

ENGEVISTA, v. 9, n. 1, p. 4-13, junho 2007 modelagem pretendida, foram selecionadas vinte e nove unidades, a saber: Manaus (AM), Santarém (PA), Macapá (AP), Belém (PA), Vila do Conde (PA), Itaqui (MA), Fortaleza (CE), Areia Branca (RN), Natal (RN), Cabedelo (PB), Recife (PE), Suape (PE), Maceió (AL), Salvador (BA), Aratu (BA), Ilhéus (BA), Barra do Riacho (ES), Vitória (ES), Rio de Janeiro (RJ), Itaguaí (RJ), Angra dos Reis (RJ), São Sebastião (SP), Santos (SP), Paranaguá (PR), São Francisco do Sul (SC), Itajaí (SC), Imbituba (SC), Porto Alegre (RS) e Rio Grande (RS).

Tendo em mente a necessidade de se manter relativamente reduzida a dimensão dos vetores de insumos e produtos nos problemas do tipo DEA, técnicos do CENTRAN, ligados ao setor portuário, definiram as quatro dimensões ou construtos que seriam suficientes para classificar os portos segundo o critério de importância no contexto de suas interações com o comércio exterior.

A primeira dimensão eleita foi a "Oferta de Infra-Estrutura" que retrata as condições físicas atuais que os portos podem oferecer ao tráfego marítimo e à movimentação de cargas. Para compor este construto foram elencadas 13 variáveis definidas por:

- $i_{1}$ : quantidade de berços especializados em contêineres. Foram considerados Berços (frente de atracação) especializados em contêineres os que possuem equipamentos apropriados para a movimentação de contêineres e que são contíguos a áreas de estocagem e vias de circulação, suficientemente capacitados, adequados e seguros para o atendimento de navios e veículos terrestres, nas operações de carregamento e descarga, dentro da área do porto organizado. Fontes: Administrações Portuárias, Anuário Estatístico da ANTAQ, Banco de Dados do CENTRAN.

- $i_{2}$ : quantidade de berços especializados em minérios. Foram considerados Berços (frente de atracação) especializados em minérios os que possuem 
equipamentos apropriados para movimentação de minérios e que são contíguos a áreas de estocagem e vias de circulação, suficientemente capacitados, adequados e seguros para o atendimento de navios e veículos terrestres, nas operações de carregamento e descarga, dentro da área do porto organizado. Com base no estudo desenvolvido pelo CENTRAN no atendimento à solicitação do Ministério dos Transportes, denominado Plano Nacional de Logística e Transportes PNLT e que pode ser encontrado na página do citado Ministério ( www.transportes.gov.br ) foram definidas as seguintes mercadorias na categoria de minérios: minério de ferro, alumina, bauxita, caulim, barrilha, manganês, calcário, coque, silício metálico e cobre. Fontes: Administrações Portuárias, Anuário Estatístico da ANTAQ, Banco de Dados do CENTRAN.

- $i_{3}$ : quantidade de berços especializados em outros granéis. Foram considerados Berços (frente de atracação) especializados em outros granéis os que possuem equipamentos apropriados para a movimentação de outros granéis e que são contíguos a áreas de estocagem e vias de circulação, suficientemente capacitados, adequados e seguros para o atendimento de navios e veículos terrestres, nas operações de carregamento e descarga, dentro da área do porto organizado. Com base no Plano Nacional de Logística de Transportes - PNLT foram definidas as seguintes mercadorias na categoria de outros granéis: grãos agrícolas, fertilizantes e sal. Fontes: Administrações Portuárias, Anuário Estatístico da ANTAQ, Banco de Dados do CENTRAN.

- $i_{4}$ : quantidade de berços especializados em neo-granéis. Foram considerados Berços (frente de atracação) especializados em neogranéis os que possuem equipamentos apropriados para a movimentação de neo-granéis e que são contíguos a áreas de estocagem e vias de circulação, suficientemente capacitados, adequados e seguros para o atendimento de navios e veículos terrestres, nas operações de carregamento e descarga, dentro da área do porto organizado. Com base no Plano Nacional de Logística de Transportes - PNLT foram definidas as seguintes mercadorias na categoria de neo-granéis: produtos siderúrgicos, veículos, celulose, mármore/granito, ferro gusa, cavaco de madeira, alumínio e bobina de papel. Fontes: Administrações Portuárias, Anuário Estatístico da ANTAQ, Banco de Dados do CENTRAN.

- $i_{5}$ : quantidade de berços com profundidade mínima de $10 \mathrm{~m}$. Berços cuja profundidade para atracação de navios é de no mínimo $10 \mathrm{~m}$. Fontes: Administrações Portuárias, Anuário Estatístico da ANTAQ.

- $i_{6}$ : profundidade mínima do acesso marítimo. Profundidade mínima verificada ao longo dos canais de acesso ao porto e das bacias de evolução e fundeio. Fontes: Administrações Portuárias, Anuário Estatístico da ANTAQ.

- $i_{7}$ : capacidade de armazenamento de contêineres. Área total especializada para armazenamento de contêineres dentro da área do porto organizado.

- $i_{8}$ : capacidade de armazenagem de minérios. Capacidade total de armazenagem de minérios dentro da área do porto organizado. Fontes: Administrações Portuárias, Anuário Estatístico da ANTAQ.

- $i_{9}$ : capacidade de armazenagem de outros granéis. Capacidade total de armazenagem de outros granéis dentro da área do porto organizado. Fontes: Administrações Portuárias, Anuário Estatístico da ANTAQ.

- $i_{10}$ : capacidade de armazenagem de neo-granéis. Capacidade total de armazenagem de neo-granéis dentro da área do porto organizado. Fontes: 
Administrações Portuárias, Anuário Estatístico da ANTAQ.

- $i_{11}$ : nível de serviço rodoviário. O nível de serviço rodoviário foi definido no PNLT como uma medida da qualidade das condições operacionais na rodovia, que procura refletir a percepção dos usuários em função de diversos fatores, tais como, velocidade e tempo de viagem, liberdade de manobras, interrupções do tráfego, segurança, conforto e conveniência. Um mesmo nível de serviço é mantido até que um volume máximo, denominado volume de serviço, seja atingido. A relação $\mathrm{V} / \mathrm{C}$ ( $V$ é a quantidade de veículos/hora, em horário de maior movimento e $\mathrm{C}$ a capacidade instalada da via), foi considerada neste trabalho apenas nos trechos de acesso aos portos das principais rodovias. $\mathrm{O}$ valor desta relação é um número entre 0 e 1 , ou seja, da situação ideal de tráfego até o uso total da capacidade instalada. Dentro deste intervalo foram consideradas 6 faixas classificadas numericamente como $0,1,2,3$, 4 e 5 .

- $i_{12}$ : quantidade de acessos ferroviários. Número de acessos ferroviários ao porto, considerando se via singela ou dupla. Esta variável é medida na escala: 0 - sem acesso, 1 linha singela e 2 - linhas duplas. Fontes: Administrações Portuárias, Anuário Estatístico da ANTAQ.

- $i_{13}$ : interferência urbana nos acessos terrestres. Existência de obstáculos (tráfego de veículos, cruzamentos, passagens de nível, invasões urbanas, etc.) ao tráfego de caminhões e trens que acessam o porto. Esta variável foi considerada em três níveis: 0 interferência intensa, 1 - interferência moderada e 2 - sem interferência. Fonte: CENTRAN.

A segunda dimensão eleita foi a de “Resultados Operacionais”, destacando a movimentação no ano base (2005), perfazendo um total de quatro variáveis:

- $o_{1}$ : quantidade movimentada de contêineres. Quantidade movimentada de contêineres no porto no ano de 2005, considerando o porto público e os terminais de uso privativo localizados na área do porto organizado. Fonte: Anuário Estatístico da ANTAQ.

- $\mathrm{O}_{2}$ : quantidade movimentada de minérios. Quantidade movimentada de minérios no porto no ano de 2005, considerando o porto público e os terminais de uso privativo localizados na área do porto organizado. Fonte: Anuário Estatístico da ANTAQ.

- $\mathrm{O}_{3}$ : quantidade movimentada de outros granéis. Quantidade movimentada de outros granéis no porto no ano de 2005, considerando o porto público e os terminais de uso privativo localizados na área do porto organizado. Fonte: Anuário Estatístico da ANTAQ.

- $\mathrm{O}_{4}$ : quantidade movimentada de neogranéis. Quantidade movimentada dos principais neo-granéis no porto no ano de 2005, considerando o porto público e os terminais de uso privativo localizados na área do porto organizado. Fonte: Anuário Estatístico da ANTAQ.

A terceira dimensão eleita foi a dos “Aspectos Geo-econômicos”, fundamental para os propósitos deste estudo e onde foram consideradas seis variáveis:

- $g_{1}$ : área de influência. Representada pela quantidade de micro regiões que a compõe, considerando as de origem e destino (pares de OD's) das principais cargas movimentadas no porto em estudo. As micro-regiões que são origem ou destino de cargas transportadas por cabotagem foram excluídas da área de influência do porto concentrador, consideradas como da área de influência do porto alimentador. Os 22 principais produtos movimentados no país, definidos no PNLT e considerados no modelo, foram: açúcar, adubo, álcool, bauxita, cana, carnes, carvão, celulose, cimento, combustível, farelos, fertilizantes, madeira, milho, minérios, oleosos, papel, petróleo, 
rocha, produtos siderúrgicos, soja e veículos.

- $g_{2}$ : produção e consumo de carga conteinerizável na área de influência. Quantidade produzida/consumida nas micro-regiões que compõem a área de influência, considerando apenas carga conteinerizável. Fonte: Banco de dados do CENTRAN.

- $g_{3}$ : produção e consumo de minérios na área de influência. Quantidade produzida/consumida de minérios nas micro-regiões que compõem a área de influência. Fonte: Banco de dados do CENTRAN.

- $g_{4}$ : produção e consumo de outros granéis na área de influência. Quantidade produzida/consumida de outros granéis nas micro-regiões que compõem a área de influência. Fonte: Banco de dados do CENTRAN.

- $g_{5}$ : produção e consumo de neogranéis na área de influência. Quantidade produzida/consumida de neo-granéis nas micro-regiões que compõem a área de influência. Fonte: Banco de dados do CENTRAN.

- $g_{6}$ : participação na corrente de comércio. Valor monetário correspondente à movimentação de cargas para exportação e importação em cada porto. Fontes: ALICEWEB da Secretaria de Comércio Exterior (SECEX), do Ministério do Desenvolvimento, Indústria e Comércio (MDIC), ano base 2005 e Anuário Estatístico da ANTAQ 2005.

A quarta e última dimensão foi eleita como sendo a de Demanda Projetada para o ano de 2011, considerando as quantidades das diferentes cargas movimentadas com base nas projeções do PNLT. Foram consideradas variáveis similares as definidas pela segunda dimensão, tomando-se aqui, os seus valores previstos:

- $p_{1}$ : quantidade movimentada de contêineres. Previsão da quantidade a ser movimentada de contêineres no porto em 2011.
- $p_{2}$ : quantidade movimentada de minérios. Previsão da quantidade a ser movimentada de minérios no porto em 2011.

- $p_{3}$ : quantidade movimentada de outros granéis. Previsão da quantidade a ser movimentada de outros granéis no porto em 2011.

- $\quad p_{4}$ : quantidade movimentada de neogranéis. Previsão da quantidade a ser movimentada de neo-granéis no porto em 2011.

\section{METODOLOGIA}

Para a hierarquização das unidades portuárias combinamos a técnica de Componentes Principais com a Análise de Envelopamento de Dados (DEA). Nesse contexto, foi definido um escore de importância utilizando a Análise de Componentes Principais a partir da matriz de correlação. Esta análise transforma o conjunto de variáveis originais em um novo conjunto de variáveis não correlacionadas, denominadas componentes principais. Nessa transformação é de particular interesse a primeira componente principal que representa a combinação linear das variáveis originais, com coeficientes com norma unitária, que possui variância máxima, isto é, que mais diferencia (separa) os portos. Esta componente é a que explica a maior parte da variabilidade observada no conjunto de dados e, tipicamente, representa a direção de maior associação com a maioria das variáveis envolvidas na classificação. A Análise de Componentes Principais a partir da matriz de correlação com o uso de variáveis padronizadas é invariante por transformações de escala e de localização. Subsequentemente faz-se uso do DEA e da noção de eficiência técnica relativa a uma fronteira de produção determinística para classificar os portos.

Os modelos de produção que levam à noção de eficiência técnica têm por objetivo avaliar a eficiência de produção de cada unidade produtora que está sendo estudada. A medida de eficiência técnica 
é uma medida entre zero e um e que responde em quanto se pode reduzir o nível utilizado dos insumos sem alterar o nível de produto. Esta ótica da medida de eficiência é conhecida como orientação a insumos.

Pode-se perguntar em quanto é viável aumentar a produção sem alterar a quantidade de insumos utilizada no processo. Esta ótica é conhecida como de orientação a produto.

Intuitivamente a primeira representa minimização de custos e a segunda maximização da receita. Se a tecnologia subjacente ao processo de produção apresentar retornos constantes à escala as orientações a insumos e produtos geram medidas equivalentes de eficiência técnica.

A aplicação dessa ordem de idéias aos portos brasileiros vai considerar um sistema de produção hipotético onde os portos são vistos, intuitivamente, por duas óticas distintas, a saber: a de um modelo de produção onde os portos "causam" o desenvolvimento, medido através dos escores dos construtos, e a de um modelo de produção em que os portos são vistos como o resultado do desenvolvimento (definido pelos construtos).

Considera-se, portanto, duas fronteiras de produção, uma com insumo unitário (a existência do porto) e produto múltiplo (as dimensões de desenvolvimento) e outra com insumo múltiplo e produto unitário.

A primeira fronteira é denominada fronteira original e a segunda fronteira invertida. O escore final de importância do porto é a média simples entre a eficiência da fronteira original e um menos a eficiência na fronteira invertida.

A abordagem com insumo/produto unitário é invariante à escala, isto é, o resultado da análise será o mesmo quer a solução seja obtida sob as hipóteses de retornos constantes ou variáveis.

A utilização de um modelo do tipo DEA em um conjunto de escores definidos por componentes principais apresenta problemas técnicos. Embora os escores finais das componentes obtidos a partir de variáveis padronizadas sejam invariantes por transformações de escala e localização isto não ocorre com a eficiência técnica. Na realidade a medida de eficiência técnica é invariante a transformações de escala, mas não a transformações de localização. Outro problema é que os escores podem ser negativos. Uma alternativa neste contexto para tornar a análise invariante por transformações de escala e localização é considerar os postos (ranks) nas componentes como respostas. Esta abordagem torna a análise robusta à presença de atipicidades e dá uma visão não paramétrica ao processo.

Será apresentada, primeiramente, a Análise de Componentes Principais concentrando a discussão nas propriedades da primeira componente, e logo após a dos modelos DEA.

A abordagem para a classificação dos portos envolve a definição, para cada dimensão, de um escore de cada porto em uma variável definida por uma combinação linear das variáveis originais convenientemente padronizadas através da subtração da média e divisão pelo desvio padrão. Esse escore é denominado construto da dimensão respectiva. Em se tratando de componentes principais, o construto da dimensão $j$ é definido pela equação:

$$
Y_{i}^{j}=\sum_{k=1}^{p_{j}} c_{i}^{j} X_{k}^{j}
$$

onde:

$Y_{i}^{j}$ é o escore do porto $i$ no construto $j$;

$c_{i}^{j}$ é o coeficiente da variável $k=1, \ldots, p_{j}$ no construto $j$ (primeiro componente principal da dimensão j); e

$x_{k}^{j}$ é o valor padronizado da variável $k$ da dimensão $j$.

Nota-se que duas abordagens são viáveis na obtenção de componentes principais. Pode-se trabalhar com a matriz de variâncias-covariâncias ou com a matriz de correlação. A padronização das variáveis implica na análise da matriz de correlação. 
A vantagem do procedimento com a matriz de correlação diz respeito a independência das unidades de medida e a colocação de todas as variáveis com mesma importância do ponto de vista da variabilidade. Embora isso pareça um pouco arbitrário, de outro modo a primeira componente principal seria dominada pelas variáveis com maior variabilidade.

Especificamente, se $R^{j}$ é a matriz de correlação entre as variáveis componentes da dimensão $j$ os coeficientes $c_{i}^{j}$ são determinados resolvendo-se o problema de otimização (Johnson e Whichern, 2007):

$$
\operatorname{Max} \alpha_{j} R^{j} \alpha_{j}^{t}, \quad \alpha_{j} \alpha_{j}^{t}=1
$$

onde:

$\alpha_{j}$ é um vetor com componentes ; e $t$ denota transposição.

Considera-se agora um processo de produção em que $p$ insumos são utilizados na produção de $s$ produtos. Se existem $n$ unidades produtoras, representa-se por $X$ a matriz $p$ por $n$ de utilização de insumos e por $Y$ a matriz $s$ por $n$ de produtos obtidos. A eficiência técnica de produção da unidade produtora i é definida pela solução do problema de programação linear Min $\theta$ sujeito as condições $\quad X \lambda \leq \theta x_{(i)}, Y \lambda \geq y_{(i)}, \lambda \geq 0$. Nesta expressão o par $\left(x_{(i)}, y_{(i)}\right)$ representa $o$ vetor de produção da unidade avaliada e $\lambda$ é um vetor de pesos de dimensão $n$. Esta formulação do DEA é conhecida como de retornos constantes à escala e é orientada para insumos. Se considerarmos 0 problema Max $\psi$ sujeito às condições $X \lambda \leq x_{(i)}, Y \lambda \geq \psi y_{(i)}, \lambda \geq 0, \quad$ tem-se a orientação para produtos sob a hipótese de retornos constantes. A medida de eficiência técnica no primeiro caso é o valor ótimo $\theta^{*}$ e no segundo caso o inverso do ótimo $\psi^{*}$. Essas medidas são coincidentes sob retornos à escala constantes (Coelli et al, 2007).
Outra questão técnica importante relacionada à técnica DEA tem a ver com as hipóteses assumidas quanto a escala de operação do processo produtivo. Pode-se demonstrar que com insumos (produtos) unitários a solução obtida independe da escala de operação do processo produtivo. Veja Lovell e Pastor (1999).

De acordo com a adaptação multicritério da metodologia do DEA procede-se ao cálculo de um "índice de desenvolvimento" para cada um dos portos. A aplicação de DEA nesse contexto será feita de duas formas. Primeiramente tomam-se os construtos como insumos e a existência dos portos como produto. Isto é, considera-se produto unitário para cada porto. Quanto maior a ineficiência do porto visto como unidade produtora em um modelo DEA voltado para a produção, maior sua importância. Assim, define-se o índice de importância do porto como sendo um menos a medida de eficiência técnica. Nessa ótica, intuitivamente, os portos são vistos como produto do desenvolvimento.

Alternativamente pode-se considerar um modelo de produção onde um insumo unitário está associado a cada porto tendo como produto múltiplo os indicadores dos construtos. Nesse caso, intuitivamente, olha-se a existência do porto como criadora do desenvolvimento. A medida de eficiência resultante é o indicador de importância do porto.

Como medida final de importância considera-se a média aritmética entre as duas medidas de importância parciais.

\section{RESULTADOS}

A Tabela 1 apresenta os resultados obtidos na Análise de Componentes Principais, as medidas de eficiência técnica com insumos e produtos unitários o escore final e a classificação final de importância. Na Análise de Componentes Principais em todas as dimensões as componentes dos autovetores apresentaram os sinais esperados. Os maiores autovalores representam 40,8\%, $46,5 \%, 55 \%$ e $50 \%$ do traço da matriz de correlação para as dimensões de infra- 
estrutura, operacional, geo-econômica e de projeção respectivamente. A esse respeito faz-se mister ressaltar que o objetivo da representação não foi o de reproduzir a variabilidade medida pelo traço.

Tabela 1: Classificação dos Portos Brasileiros segundo suas posições relativas nas dimensões de Infra-estrutura, Operacional, Geo-econômica e de Projeção para 2011. Dea_1 é a fronteira original e Dea_2 a fronteira invertida. Var é a média de Dea_1 e 1Dea_2 e R_var a classificação em ordem decrescente de importância.

\begin{tabular}{|c|c|c|c|c|c|c|c|c|}
\hline Porto & R_infra & R_opera & R_geo & R_proj & Dea_1 & Dea_2 & Var & R_Var \\
\hline Manaus & 0,034 & 0,345 & 0,310 & 0,483 & 0,483 & 1,000 & 0,242 & 6 \\
\hline Santarém & 0,276 & 0,448 & 0,483 & 0,517 & 0,517 & 0,973 & 0,272 & 7 \\
\hline Macapá & 0,069 & 0,379 & 0,655 & 0,690 & 0,690 & 0,996 & 0,347 & 15 \\
\hline Belém & 0,310 & 0,207 & 0,241 & 0,345 & 0,345 & 0,987 & 0,179 & 4 \\
\hline Vila do & 0,759 & 0,517 & 0,621 & 0,207 & 0,759 & 0,969 & 0,395 & 19 \\
\hline Itaqui & 0,724 & 0,724 & 0,793 & 0,655 & 0,793 & 0,901 & 0,446 & 21 \\
\hline Fortaleza & 0,552 & 0,483 & 0,448 & 0,448 & 0,552 & 0,967 & 0,293 & 10 \\
\hline Areia Branca & 0,138 & 0,690 & 0,586 & 0,103 & 0,690 & 0,999 & 0,346 & 14 \\
\hline Natal & 0,207 & 0,069 & 0,069 & 0,034 & 0,207 & 1,000 & 0,104 & 1 \\
\hline Cabedelo & 0,483 & 0,034 & 0,034 & 0,241 & 0,483 & 1,000 & 0,242 & 6 \\
\hline Recife & 0,517 & 0,414 & 0,345 & 0,586 & 0,586 & 0,980 & 0,303 & 11 \\
\hline Suape & 0,793 & 0,586 & 0,517 & 0,621 & 0,793 & 0,951 & 0,421 & 20 \\
\hline Maceió & 0,345 & 0,552 & 0,379 & 0,414 & 0,552 & 0,973 & 0,290 & 9 \\
\hline Salvador & 0,448 & 0,655 & 0,552 & 0,379 & 0,655 & 0,943 & 0,356 & 16 \\
\hline Aratu & 0,655 & 0,172 & 0,414 & 0,276 & 0,655 & 0,988 & 0,334 & 13 \\
\hline Ilhéus & 0,103 & 0,310 & 0,172 & 0,552 & 0,552 & 1,000 & 0,276 & 8 \\
\hline $\begin{array}{c}\text { Barra do } \\
\text { Riacho }\end{array}$ & 0,690 & 0,621 & 0,690 & 0,310 & 0,690 & 0,956 & 0,367 & 17 \\
\hline Vitória & 0,862 & 0,862 & 0,862 & 0,897 & 0,897 & 0,863 & 0,517 & 24 \\
\hline $\begin{array}{l}\text { Rio de } \\
\text { Janeiro }\end{array}$ & 0,897 & 0,897 & 0,828 & 0,793 & 0,897 & 0,854 & 0,522 & 25 \\
\hline Itaguaí & 0,828 & 0,759 & 0,966 & 0,759 & 0,966 & 0,889 & 0,539 & 26 \\
\hline $\begin{array}{c}\text { Angra dos } \\
\text { Reis }\end{array}$ & 0,621 & 0,138 & 0,138 & 0,069 & 0,621 & 0,996 & 0,313 & 12 \\
\hline $\begin{array}{c}\text { São } \\
\text { Sebastião }\end{array}$ & 0,241 & 0,103 & 0,103 & 0,172 & 0,241 & 0,999 & 0,121 & 2 \\
\hline Santos & 1,000 & 1,000 & 1,000 & 1,000 & 1,000 & 0,446 & 0,777 & 29 \\
\hline $\begin{array}{c}\text { Paranaguá } \\
\text { São }\end{array}$ & 0,931 & 0,966 & 0,897 & 0,966 & 0,966 & 0,715 & 0,626 & 28 \\
\hline $\begin{array}{c}\text { Francisco do } \\
\text { Sul }\end{array}$ & 0,586 & 0,828 & 0,724 & 0,828 & 0,828 & 0,895 & 0,467 & 22 \\
\hline Itajaí & 0,414 & 0,793 & 0,759 & 0,862 & 0,862 & 0,906 & 0,478 & 23 \\
\hline Imbituba & 0,379 & 0,276 & 0,207 & 0,724 & 0,724 & 0,987 & 0,369 & 18 \\
\hline Porto Alegre & 0,172 & 0,241 & 0,276 & 0,138 & 0,276 & 0,995 & 0,141 & 3 \\
\hline Rio Grande & 0,966 & 0,931 & 0,931 & 0,931 & 0,966 & 0,757 & 0,605 & 27 \\
\hline
\end{tabular}

A Figura 1 ilustra a distribuição do escore final de avaliação. Observamos que os testes de normalidade usuais de Shapiro-Wilks, Smirnov - Kolmogorov, Cramer - von Mises e Anderson Darling não são significantes com pvalores de $63,7 \%$, ,>15\%, $>25 \%$ e $>25 \%$, respectivamente. Muito provavelmente a distribuição do escore pode ser ajustada ENGEVISTA, v. 9, n. 1, p. 4-13, junho 2007 por uma normal truncada em $(0,1)$. A única observação potencialmente atípica é a do porto de Santos, que se sobressai na medida de importância.

O diagrama de caixa da Figura 1 ilustra o resumo de 5 números definido pelos extremo inferior, primeiro quartil, mediana, terceiro quartil e extremo superior, eliminando-se atípicos. Esses 
valores são 0,104, 0,276, 0,347, 0,467 e 0,626 , respectivamente. Do ponto de vista da aplicação de recursos nos portos é de interesse a divisão da população dos portos pelos quartis. O grupo acima do terceiro quartil é definido pelos portos de Santos, Paranaguá, Rio Grande, Itaguaí, Rio de Janeiro, Vitória e Itajaí. São Francisco do Sul é a fronteira do quartil
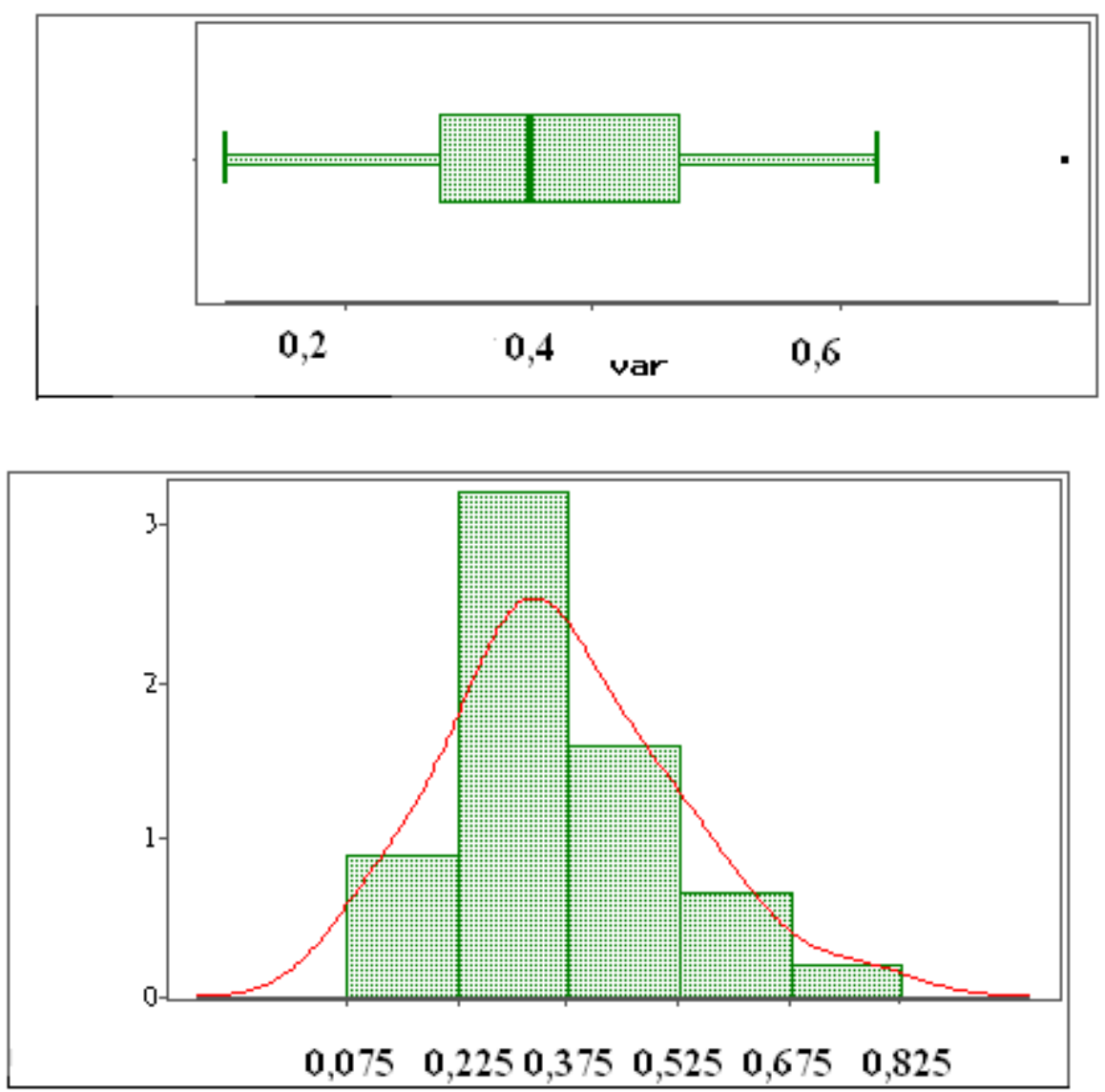

Figura 1: Distribuição do escore final de classificação (Var). Estimador não paramétrico da densidade superposto ao histograma.

\section{RESUMO E CONCLUSÕES}

Classificaram-se os portos brasileiros por meio de um critério de importância que combina a Análise Estatística Multivariada e a Análise de Envelopamento de Dados. Quatro dimensões foram consideradas para a caracterização do conceito de importância: Infra-estrutura, Operacional, Geo-econômica e de Previsão Operacional em 2011. Essas quatro dimensões compreendem um total de 29 variáveis reduzidas a 4 via a Análise de Componentes Principais. Em cada dimensão considerou-se 0 posto normalizado do escore na primeira componente principal como medida de insumo (produto) em um modelo DEA multicritério com produto (insumo) unitário. A análise é invariante por transformações de escala e localização e robusta relativamente à presença de atipicidades no contexto DEA.

A título de ilustração, na dimensão Infra-estrutura, a variável de maior representatividade é a "Disponibilidade de Berços de Atracação com profundidade igual ou maior que 10 metros", com coeficiente de correlação de 94,28\%. Por sua vez na dimensão Resultados Operacionais, as variáveis "Movimentação de Contêineres" e "Movimentação de Outros Granéis", com coeficientes de $96,2 \%$ e $94,46 \%$ respectivamente, são as mais influentes. Na dimensão Aspectos Geo-econômicos, as variáveis "Produção/Consumo de Outros Granéis”, com 90,66\% e "Participação na Corrente do Comércio", 
com $90,48 \%$ são as que mais influenciam. Por sua vez, na dimensão "Demanda Projetada - 2011" a variável mais influente é a relativa a "Movimentação de Contêineres" com 90,12\% de coeficiente de correlação com a dimensão.

Do ponto de vista da aplicação de recursos no setor portuário, selecionados os portos que mais atendem ao objetivo de apoio ao comércio exterior bem como os projetos já agendados ou em desenvolvimento nessas unidades, sugere-se maiores injeções de recursos naqueles que trarão maior benefício econômico para o Brasil. Entende-se aqui, neste contexto, que o construtos considerados para a classificação sejam representativos.

Nesse sentido, o ente portuário, enquanto elo relevante da cadeia logística de transporte é fortemente condicionado pelo contexto econômico em que se insere, como se pode observar pela predominância, nas primeiras posições, dos portos localizados no sul-sudeste brasileiro, indiscutivelmente a região de economia mais consolidada do País. Particularmente, o Porto de Santos apresentou os melhores resultados nas quatro dimensões consideradas demonstrando, assim, a sua preponderância no atual quadro portuário nacional, destacando-se pelo número de berços especializados, grande movimentação de cargas atualmente e previstas, que o mantém na liderança da movimentação de contêineres em 2011, além de apresentar uma participação de 43,03\% na Corrente de Comércio Brasileira no ano base do estudo.

À luz dos pressupostos do modelo adotado e do cenário portuário atual do País, conclui-se pela significativa consistência e representatividade do resultado obtido.

\section{REFERÊNCIAS BIBLIOGRÁFICAS}

COELLI, T. J; RAO, D. S., O’DONNEL,C. CJ.; BATTESE,G. E. An
Introduction to Efficiency and Productivity Analysis, 2ed. Springer, 2007.

COOK, W.D. Qualitative data in DEA. In: COOPER, W.C.; SEIFORD, L.M.; ZHU, J. (eds) Handbook on Data Envelopment Analysis. Kluwer, 2004.

DE KOEIJER, T.J.; WOSSINK, G.A.A.; STRUIK, P.C.; RENKEMA, J.A. Measuring agricultural sustainability in terms of efficiency: the case of Dutch sugar beet growers. Journal of Environmental Management, v. 66, p. 9-17, 2002.

JOHNSON R. A.; WICHERN D. W., Applied Multivariate Analysis, 6ed. Prentice Hall, 2007.

LETA, F.R.; SOARES DE MELLO, J.C.C.B.; GOMES, E.G.; ÂNGULO MEZA, L. Métodos de melhora de ordenação em DEA aplicados à avaliação estática de tornos mecânicos. Investigação Operacional, v. 25, n. 2, p. 229-242, 2005.

LOVELL, C.A.K.; PASTOR, J.T. Radial DEA models without inputs or without outputs. European Journal of Operational Research, v. 118, n. 1, p. 46-51, 1999.

PLANO NACIONAL DE LOGÍSTICA E TRANSPORTES - PNLT (2007).

SAATY, T. L. The Analytic hierarchy process. RWS, 1994.

SOUZA, G.S. The law of categorical judgment revisited. Brazilian Journal of Probability and Statistics, v. 16, p. 123140, 2002.

THOMPSON, R.G.; SINGLETON Jr., F.D.; THRALL, R.M.; SMITH, B.A. Comparative evaluation for locating a high-energy physics lab in Texas. Interfaces, v.16, p. 35-49, 1986. 